\title{
ON THE ROLE OF ENTEROCOCCI IN THE BLOODSTREAM: RESULTS OF A SINGLE-CENTER, RETROSPECTIVE, OBSERVATIONAL STUDY AT A GERMAN UNIVERSITY HOSPITAL
}

\author{
Hagen Frickmann ${ }^{1,2, *}$, Kerstin Köller ${ }^{2}$, Irina Veil ${ }^{2}$, Mirjam Weise ${ }^{2}$, Alicja Ludyga ${ }^{3}$, Norbert Georg Schwarz ${ }^{4}$, \\ Philipp Warnke ${ }^{2}$, Andreas Podbielski \\ ${ }^{1}$ Department of Tropical Medicine at the Bernhard Nocht Institute, Bundeswehr Hospital Hamburg, Hamburg, Germany \\ ${ }^{2}$ Institute for Medical Microbiology, Virology and Hygiene, University Medicine Rostock, Rostock, Germany \\ ${ }^{3}$ aLTRan Ltd., Munich, Germany \\ ${ }^{4}$ Infectious Disease Epidemiology, Bernhard Nocht Institute for Tropical Medicine Hamburg, Hamburg, Gemany
}

Received: September 5, 2017; Accepted: September 7, 2017

This study assesses the clinical relevance of vancomycin-susceptible enterococci in bacteremic patients and compares it with bacteremia due to Staphylococcus aureus and Escherichia coli.

During a 5-year-study interval, clinical and diagnostic features of patients with enterococcal bacteremia were compared to those of patients with $E$. coli or $S$. aureus bacteremia. Each patient was only counted once per hospital stay.

During the 5-year study interval, data from 267 patients with enterococcal bacteremia and from 661 patients with bacteremia due to $E$. coli or $S$. aureus were evaluated. In spite of a comparable risk of death, patients with enterococci more frequently needed catecholamines and invasive ventilation. Furthermore, enterococci were more frequently associated with a mixed bacterial flora in bloodstream infections. While fatal sepsis due to E. coli and S. aureus was associated with typical shock symptoms, this association was not confirmed for enterococci.

Although enterococcal bacteremia is associated with a risk of dying comparable to that with bacteremia due to $E$. coli and $S$. aureus, a lower pathogenic potential of enterococci in bloodstream has to be acknowledged. Enterococci in the bloodstream are more likely to be an epiphenomenon of impending death than its major cause.

Keywords: enterococci, bloodstream infection, fatal outcome, Enterococcus spp., blood culture

\section{Introduction}

On human mucous membranes, particularly in the gut, enterococci usually persist as harmless to useful colonizers. A pathological role for enterococci is well established for urinary tract infections [1], in bacterial endocarditis [2], or in cases of translocation into primarily sterile compartments $[3,4]$. Prophylaxis with combinations of cephalosporins and metronidazole works well during surgical operations on the open gut $[5,6]$, although enterococci are not susceptible to these antibiotics. This is regarded as an argument for the low etiological relevance of enterococci in abdominal infections [5]. In corpses, in contrast, enterococci spread readily throughout the body of the deceased after the breakdown of the gut-blood barrier [7] and drive their decomposition. In line with a presumably low pathogenicity, death after enterococcal infections is mainly observed in immunocompromised patients [8-11].

With respect to this overall low relevance of enterococci in deep-seated infections, this study was undertaken to determine their etiological relevance in bloodstream infections, which has not yet been satisfactorily assessed.

\footnotetext{
* Corresponding author: Hagen Frickmann; Department of Tropical Medicine at the Bernhard Nocht Institute, German Armed Forces Hospital of Hamburg, Bernhard Nocht Str. 74, 20359 Hamburg, Germany; Phone: 0049-40-6947-28700;

Fax: 0049-40-6947-28709; E-mail: Frickmann@bni-hamburg.de
}

This is an open-access article distributed under the terms of the Creative Commons Attribution-NonCommercial 4.0 International License (https://creativecommons.org/licenses/by-nc/4.0/), which permits unrestricted use, distribution, and reproduction in any medium for non-commercial purposes, provided the original author and source are credited, a link to the CC License is provided, and changes - if any - are indicated. 
Clinical and diagnostic features of Enterococcus spp.associated bacteremia were assessed and compared with those of bacteremia due to Staphylococcus aureus (both methicillin-susceptible and -resistant) and Escherichia coli, both of which are well-established causative agents of sepsis $[12,13]$.

We have set up the working hypothesis that, unlike sepsis caused by established bacterial pathogens, enterococcal bacteremia is primarily encountered in two groups of patients: One is comparably healthy and suffers from transient bacteremia potentially associated with surgical procedures or inflamed mucous membranes. The other consists of pre-mortal patients with a disrupted gut-blood barrier allowing easy translocation of gut-inhabiting bacteria like enterococci from the gut into the bloodstream. To test this hypothesis, the following severity indicators were compared: automated ventilation, catecholamine requirement, and death of patients bacteremic for one of the three species. Identification of polymicrobial bacteremia was used as a criterion for translocation events in severely ill patients.

\section{Methods}

The study was designed as a retrospective observational study and carried out at the University Hospital of Rostock, Germany, covering a study interval from 2007 to 2011.

All patient samples were collected and processed according to the instructions of the accredited (DIN EN ISO 15189) Institute for Medical Microbiology, Virology and Hygiene of the Rostock University Hospital. The resulting data were analyzed with the SwissLab software (version 2.12.2.008848; SwissLab Ltd., Berlin, Germany) of the laboratory.

\section{Assessment of the frequency of enterococci in blood cultures at the study site}

The data from the 20 most frequent species isolated from blood culture bottles (BacT/ALERT SA/SN/FA/FN/PF, bioMérieux, Nürtingen, Germany) at the Institute for Medical Microbiology, Virology and Hygiene were used for comparison. Data sets were extracted from the SwissLab laboratory software and analyzed using the HyBase software version 6.1508.1 (Tieto Germany Ltd., Regensburg, Germany). Mean values were calculated from the data from the study interval.

\section{Inclusion and exclusion criteria}

For a more refined analysis, data from patients of the University Hospital Rostock suffering from Enterococcus spp. and/or E. coli and/or $S$. aureus (methicillin-susceptible or -resistant) bacteremia in the years 2007 to 2011 were included in the study. In the following sections, data sets from $S$. aureus and E. coli were occasionally combined for statistical reasons and are then referred to as "nonenterococci". Each bacterial species was counted only once per patient and hospital admission to avoid analyzing copy strains.

\section{Assessment of clinical data}

Available clinical data were retrospectively assessed from the medical records of the included patients. The assessment was performed anonymously to ensure the patients' rights to privacy. As well as age, gender, and death of patients during the hospital stay, a total of 20 clinical and laboratory features per patient were assessed. The assessment comprised differentiation of pure and mixed bacterial culture; surgical interventions in general and abdominal surgery in particular; presence of wounds, urinary catheters, and stomata; body temperature; blood pressure according to Riva-Rocci (RR) [14]; pulse rate; leukocyte count; C-reactive protein levels; procalcitonin levels; detection of leukocytes and bacteria in urine samples; need for treatment in intensive care units (ICUs); catecholamine administration or invasive ventilation at the ICU; the presence of bacterial isolates from bronchoalveolar lavage; sepsis as the main diagnosis; and administration of antibiotic therapy at the time of sample acquisition.

The results were adjusted to remarkable findings as depicted and defined in Table 2 from the Results section. All data were included in a SAS database, version 9.4 (SAS Institute Inc., Cary, NC, USA), which was also used for all statistical assessments as described below.

\section{Comparison of the enterococcus group and the non- enterococcus group regarding hard endpoints}

Hard endpoints for patients with enterococcal or nonenterococcal bacteremia were defined as need for invasive ventilation, need for catecholamine administration, and death at any time point in the hospital stay after the diagnosis of bacteremia. The data from the enterococcus and the nonenterococcus groups were compared with the $\chi^{2}$ test.

\section{Assessment of the enterococcus group and the non- enterococcus group for single species or mixed bacterial species in blood cultures}

In the first step, differences between the frequencies of mixed-species bacterial culture in the enterococcus group and the nonenterococcus group were assessed based on odds ratios. In the second step, the same calculation was repeated for deceased patients only. 
Comparison of factors associated with survival and death for the enterococcus group and the nonenterococcus group

To identify factors associated with death specifically due to enterococcal bacteremia, all recorded clinical features were analyzed for associations with survival and death in both the enterococcus group and the nonenterococcus group. Taken together, this assessment should discriminate whether dying patients from the enterococcus group were more severely affected than patients from the nonenterococcus group. Furthermore, it should distinguish the extent of differences in clinical illness between dying and surviving patients in the two bacteremic groups.

A logistic regression (model: binary logit; optimization: Fischer's scoring) was performed to indicate the impact of score parameters on the fatal outcome for patients with enterococcal and nonenterococcal bacteremia. Then, an adapted logistic regression (model: binary logit; optimization: Fischer's scoring) with stepwise elimination was added to indicate only significant variables and two-way interactions regarding fatal outcome for patients with enterococcal and nonenterococcal bacteremia. Significance was assessed by $\chi^{2}$ testing.

Comparison of factors associated with other hard endpoints in the enterococcus group and the nonenterococcus group

The initially chosen hard endpoint parameters of death, need for catecholamine administration, and need for invasive ventilation were analyzed for independence. After reciprocal dependence of the three parameters had been proven by crosstabs (data not shown), logistic regression (model: binary logit; optimization: Fischer's scoring) with stepwise elimination was performed to indicate only significant variables and two-way interactions regarding the composite outcome "need for catecholamines and need for invasive ventilation and fatal outcome" for patients with enterococcal and nonenterococcal bacteremia. Significance was assessed by $\chi^{2}$ testing.

\section{Ethics statement}

Ethical clearance for the study, including the anonymized retrospective assessment of patients' data from their medical records without informed consent of the patients, was approved by the ethics committee of the University Medicine Rostock (study registration number: A 2015-0078) in accordance with relevant guidelines and regulations.

\section{Results}

\section{Frequency of enterococci in blood cultures at the study site}

In the list of the 20 most frequently isolated species from blood cultures at the University Hospital Rostock during the study period (Supplementary Material 1), Enterococcus faecium was at position no. 6 and Enterococcus faecalis at position no. 8. Next to typical skin contaminants such as coagulase-negative staphylococci and Propionibacterium spp., E. coli and $S$. aureus were the two most frequently isolated species with probable or at least presumptive etiological relevance for severe deep-seated infections or sepsis (Supplementary Material 1).

\section{Included and excluded patients and samples}

During the study interval, 32,394 blood culture bottles were assessed at the University Medicine Rostock. Adjusted for samples from the same patient during the same hospital stay, there were 3992 patients with one or more positive blood cultures and 12,266 patients with negative blood cultures. The distribution of analyzed blood culture samples as assessed by the laboratory statistics is depicted in Table 1.

Table 1. Statistics of assessed blood culture samples during the study interval from 2007 to 2011 at the University Medicine Rostock

\begin{tabular}{|c|c|c|c|c|c|c|}
\hline Groups & $2007-2011$ & 2007 & 2008 & 2009 & 2010 & 2011 \\
\hline Assessed blood culture samples (total) & 32394 & 6324 & 6165 & 6092 & 6924 & 6889 \\
\hline Assessed blood culture samples (corrected by copy strains) & 16258 & 3020 & 3078 & 3209 & 3433 & 3518 \\
\hline Positive blood culture samples (total) & 5640 & 1053 & 1134 & 1036 & 1177 & 1240 \\
\hline Positive blood culture samples (corrected by copy strains) & 3992 & 758 & 777 & 750 & 810 & 897 \\
\hline Negative blood culture samples (total) & 26754 & 5271 & 5031 & 5056 & 5747 & 5649 \\
\hline Negative blood culture samples (corrected by copy strains) & 12266 & 2262 & 2301 & 2459 & 2623 & 2621 \\
\hline Detected enterococci (total) & 567 & 133 & 98 & 92 & 140 & 104 \\
\hline Detected enterococci (corrected by copy strains) & 369 & 88 & 66 & 63 & 86 & 66 \\
\hline Included patients with enterococci into the assessment & $267^{*}$ & 48 & 40 & 30 & 49 & 41 \\
\hline Detected Enterococcus faecalis (total) & 221 & 48 & 34 & 46 & 55 & 38 \\
\hline
\end{tabular}


Table 1. (cont'd)

\begin{tabular}{|c|c|c|c|c|c|c|}
\hline Groups & $2007-2011$ & 2007 & 2008 & 2009 & 2010 & 2011 \\
\hline Detected Enterococcus faecalis (corrected by copy strains) & 147 & 30 & 26 & 31 & 30 & 30 \\
\hline Included patients with Enterococcus faecalis into the assessment & $112^{* *}$ & 17 & 18 & 16 & 13 & 22 \\
\hline Detected Enterococcus faecium (total) & 312 & 75 & 62 & 37 & 77 & 61 \\
\hline Detected Enterococcus faecium (corrected by copy strains) & 196 & 51 & 39 & 25 & 50 & 31 \\
\hline Included patients with Enterococcus faecium into the assessment & $141^{\dagger}$ & 29 & 22 & 11 & 35 & 16 \\
\hline Detected S. aureus (including MRSA, total) & 650 & 84 & 135 & 126 & 165 & 140 \\
\hline Detected $S$. aureus (including MRSA, corrected by copy strains) & 363 & 51 & 77 & 70 & 84 & 81 \\
\hline Included patients with $S$. aureus (including MRSA) into the assessment & $286^{*}$ & 30 & 52 & 49 & 49 & 61 \\
\hline Detected MSSA (total) & 434 & 49 & 84 & 98 & 107 & 96 \\
\hline Detected MSSA (corrected by copy strains) & 275 & 37 & 54 & 57 & 67 & 60 \\
\hline Included patients with MSSA into the assessment & $219^{\S}$ & 21 & 35 & 38 & 35 & 44 \\
\hline Detected MRSA (total) & 216 & 35 & 51 & 28 & 58 & 44 \\
\hline Detected MRSA (corrected by copy strains) & 88 & 14 & 23 & 13 & 17 & 21 \\
\hline Included patients with MRSA into the assessment & $67^{\natural}$ & 9 & 17 & 11 & 4 & 17 \\
\hline Detected E. coli (total) & 659 & 105 & 132 & 119 & 135 & 168 \\
\hline Detected E. coli (corrected by copy strains) & 463 & 75 & 91 & 87 & 87 & 123 \\
\hline Included patients with $E$. coli into the assessment & $375^{\#}$ & 42 & 57 & 54 & 46 & 94 \\
\hline
\end{tabular}

${ }^{*}$ Missing documentation of isolation year for 59 data sets

${ }^{* *}$ Missing documentation of isolation year for 26 data sets

${ }^{\dagger}$ Missing documentation of isolation year for 28 data sets

*Missing documentation of isolation year for 55 data sets

${ }^{\S}$ Missing documentation of isolation year for 46 data sets

"Missing documentation of isolation year for 9 data sets

"Missing documentation of isolation year for 82 data sets

Over the 5-year-interval of the study, 267 patients with enterococci in blood cultures, comprising 121 E. faecalis, 141 E. faecium, 4 E. avium, 1 E. casseliflavus, 1 E. cecorum, and 6 not further characterized Enterococcus spp., and 661 patients with nonenterococci, comprising 375 E. coli, 219 methicillin-susceptible S. aureus, and 67 methicillin-resistant $S$. aureus (MRSA), respectively, were identified and included in the study (Table 1). Each patient was counted only once per hospital stay, irrespective of the number of positive blood cultures or the time between them. As also detailed in Table 2, the coverage of data assessment of the affected patients ranged between onehalf and three-quarters of the total number of patients with bacteremia due to enterococci, E. coli, or $S$. aureus. The incompleteness is due to the fact that not all patient files were assessable for logistic reasons.

The mean age of patients with enterococcal bacteremia was 63.8 years; that of patients with nonenterococcal bacteremia was 65.5 years. Among the individuals with enterococcal bacteremia, $67 \%$ were male; among the ones with nonenterococcal bacteremia, $56 \%$ were male.

\section{Assessment of clinical data}

Clinical characteristics were compared for enterococcal and nonenterococcal bacteremia (Table 2), ensuring suf- ficiently high patient numbers for statistical assessments as described in the following.

\section{Comparison of the enterococcus group and the non- enterococcus group regarding hard endpoints}

Considering the two endpoints, i.e., need for mechanical ventilation and need for catecholamines, the risk was considerably increased in patients with enterococcal bacteremia in comparison with patients with $E$. coli or $S$. aureus bacteremia. The risk of dying, however, was identical in both groups (Table 3). There were no relevant differences between E. faecium- and E. faecalis-associated bacteremia.

Assessment of the enterococcus group and the nonenterococcus group for one or more bacterial species in blood cultures

The presence of more than one bacterial species in the blood culture medium was significantly more frequent in patients with enterococcal bacteremia than in patients with bacteremia due to $E$. coli or $S$. aureus. If only deceased patients were analyzed, the significance of this difference disappeared (Table 4). Again, there were no detectable differences between E. faecalis and E. faecium. 


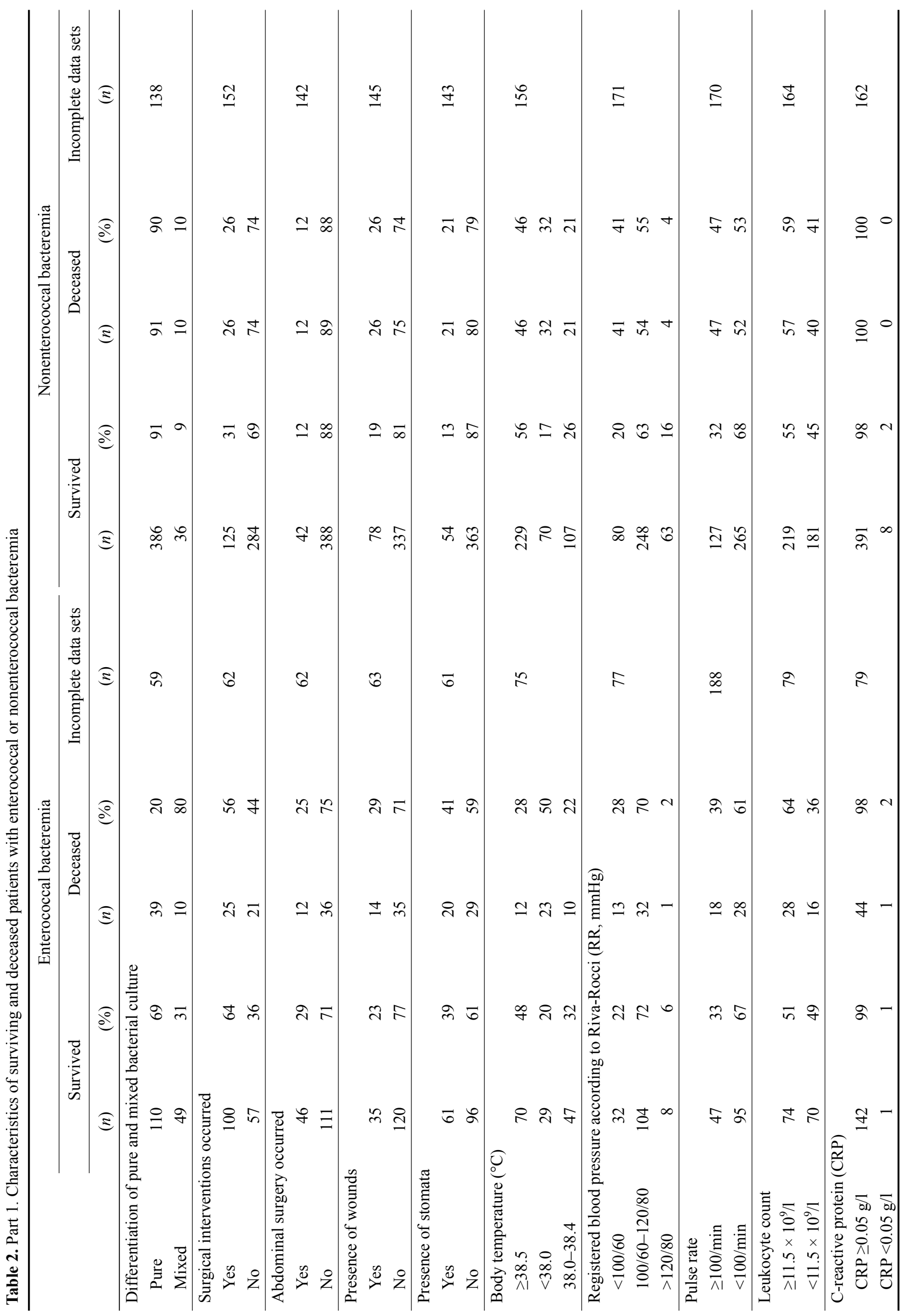

European Journal of Microbiology and Immunology 


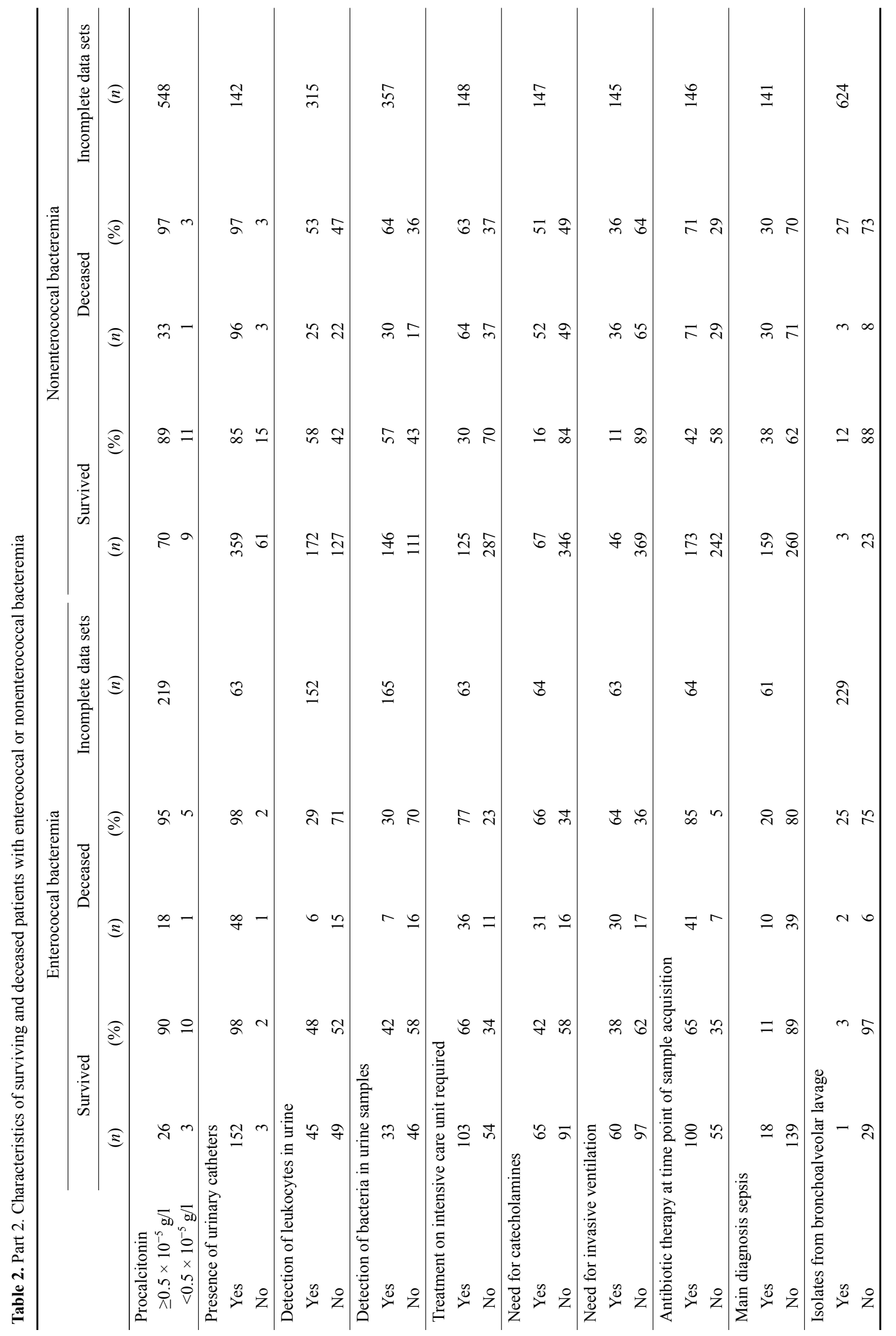




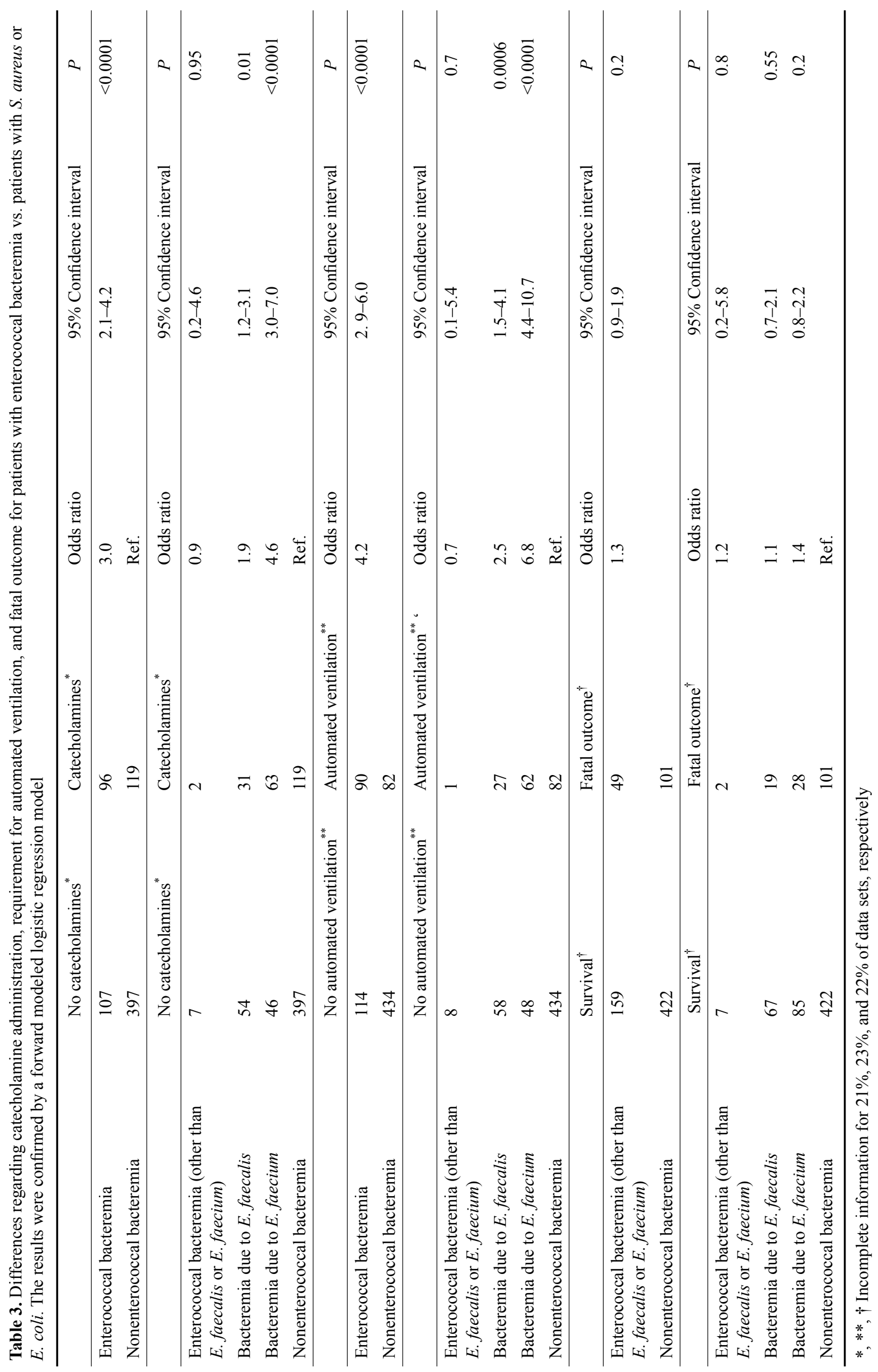



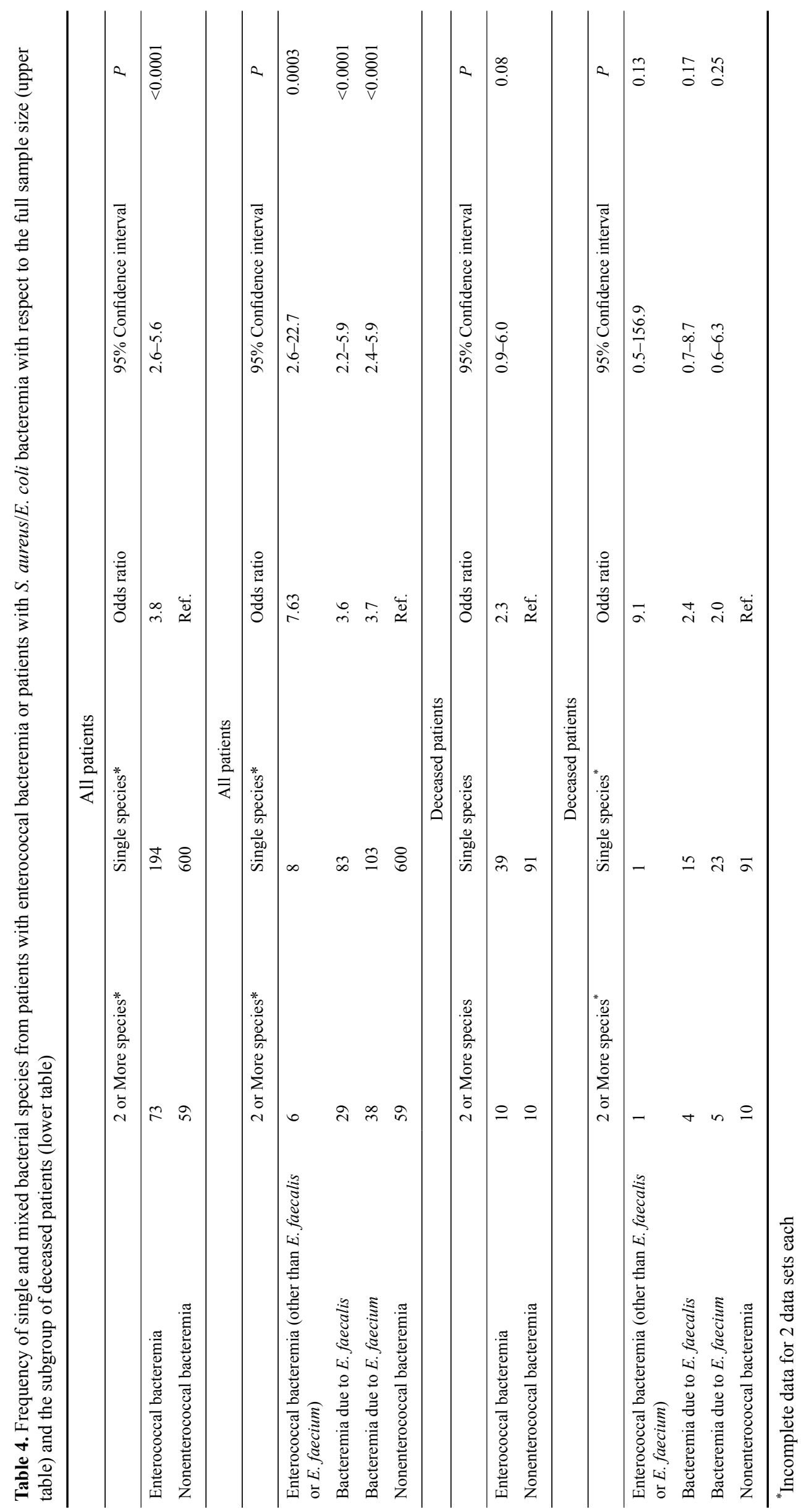

European Journal of Microbiology and Immunology 


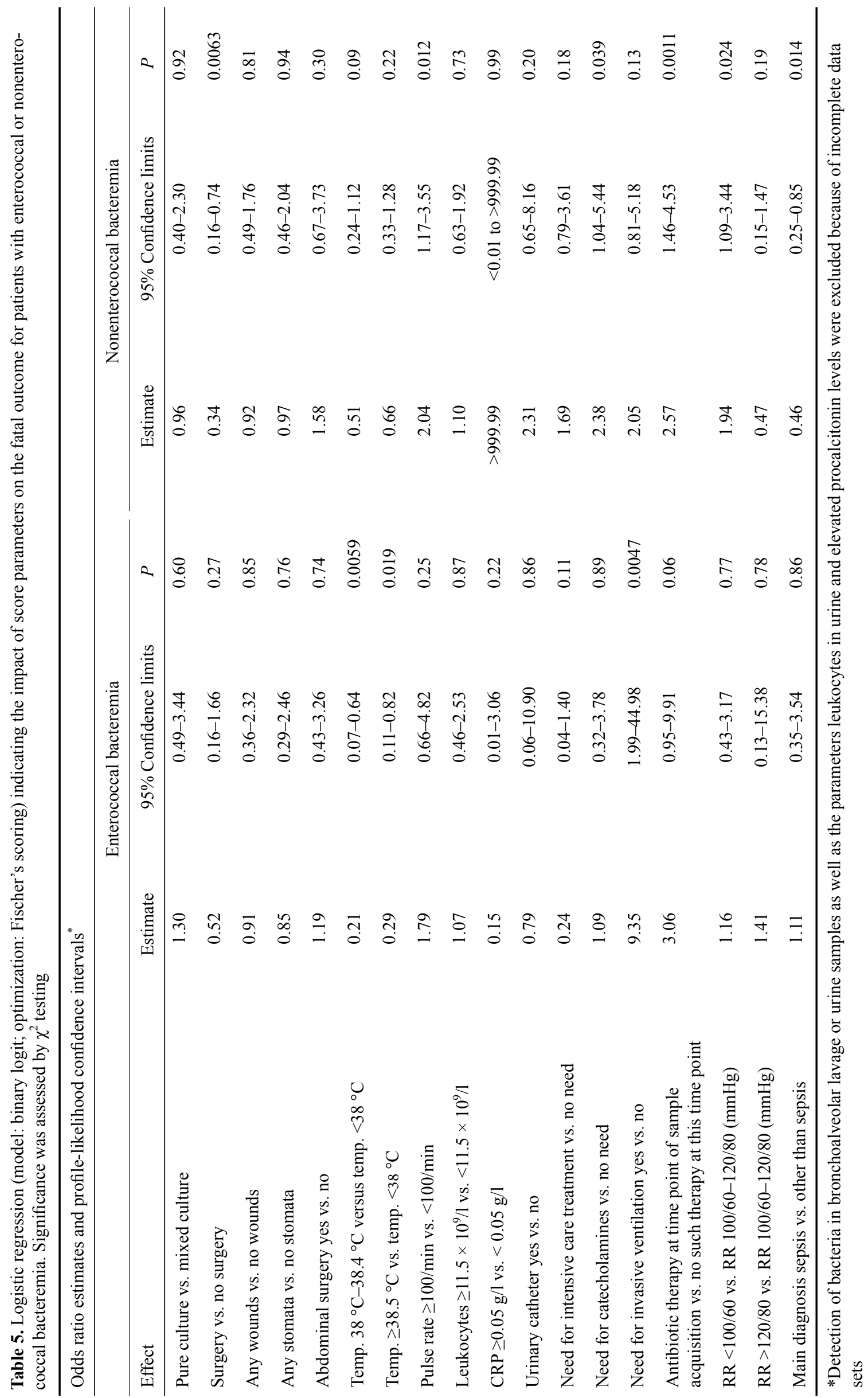


The species distribution of the bacteria accompanying enterococci, $E$. coli, and $S$. aureus in mixed species blood cultures showed an inconclusive pattern (Supplementary Material 2). The low numbers of observed isolates did not allow for statistical analysis. However, enterococci were more frequently associated with coagulase-negative staphylococci than S. aureus or E. coli.

\section{Factors associated with survival or death of patients with enterococcal or nonenterococcal bacteremia}

Logistic regression (Table 5) and logistic regression with stepwise elimination of factors (Supplementary Material 3) indicated that patients with bacteremia due to $E$. coli and $S$. aureus were more likely to die in case of tachycardia with a pulse rate $\geq 100 / \mathrm{min}$, low blood pressure (RR) of $<100 / 60$, and need for catecholamines. For enterococcal bacteremia, this association was not observed.

Comparison of factors associated with a combined endpoint comprising "need for invasive ventilation, need for catecholamines, and death" in patients with enterococcal or nonenterococcal bacteremia

When analyzed in cross tables, the three endpoints "need for invasive ventilation, need for catecholamines, and death" showed reciprocal dependence (data not shown). Therefore, an additional analysis that regarded the three parameters as a single composite endpoint was performed. Specifically for patients with $E$. coli and $S$. aureus bacteremia, the presence of stomata and intravenous catheters was associated with an increased risk. Specifically for patients with enterococcal bacteremia, fever combined with the presence of stomata was the only identified risk factor. Associations of shock parameters were no longer observed (Supplementary Material 4).

\section{Discussion}

The study was performed to address the predisposing factors for and etiological relevance of enterococci in blood culture. Bacteremia due to E. coli and $S$. aureus, a likely cause of sepsis $[12,13]$, was chosen as a reference for the comparison. As described for other European hospitals $[15,16]$, E. faecium quantitatively dominated in comparison to the therapeutically less problematic E. faecalis. This is in contrast to other regions such as Australia, where E. faecalis dominates [17]. Based upon data from the German National Surveillance System, an annual incidence of about 30,000 nosocomial E. faecium infections has been calculated, comprising $13.2 \%$ vancomycin-resistant isolates [18], demonstrating the importance of vancomycinsusceptible strains.

The study produced several main results. First of all, patients with enterococcal bacteremia are typically more severely ill than patients with typical causative agents of sepsis like E. coli and $S$. aureus in their bloodstream. In spite of a comparable risk of death, patients with enterococci more frequently need catecholamines and invasive ventilation. In addition, enterococcal bacteremia was more frequently associated with at least one additional bacterial species in the bloodstream. Finally, fatal sepsis due to $E$. coli and $S$. aureus is usually associated with shock symptoms such as tachycardia, hypotonia, and need for catecholamines, whereas this association was not identified for the enterococci. The synopsis of these results suggests that severely ill patients more frequently suffer from enterococcal bacteremia, while patients who are still able to show a systemic inflammatory reaction resulting in shock are more likely to die from sepsis due to E. coli and S. aureus.

Data on the risk for bacteremia with vancomycin-susceptible enterococci are still rare [9]. In particular, comparisons with the features of infections due to nonenterococcal causes of sepsis are usually absent. Bacteremia due to enterococci is usually hospital acquired, with urinary tract infections, intra-abdominal infections, and infective endocarditis as the major sources [10]. In India, the rate of enterococcal bacteremia has been estimated at 25.4 episodes per 1000 admissions in trauma patients [19]. Preliminary recent data suggest increased mortality in cases of repeated detection of positive blood cultures with enterococci, in particular in case of prolonged bloodstream infections lasting more than 6 days [20].

This study implies that enterococcal bacteremia and E. coli and $S$. aureus bacteremia display identical risks of dying, being $18.4 \%$ and $19.3 \%$ of the respective patients. This mortality matches that in a Polish study on enterococcal bacteremia, which indicated a 14-day mortality of $18.1 \%$ [21]. In pediatric patients with enterococcal bacteremia after hematopoietic stem cell transplantation, a 30 -day mortality of $20 \%$ has been reported irrespective of vancomycin susceptibility or resistance traits of the strains [9]. In a Danish assessment, 30-day mortality of $21.4 \%$ due to E. faecalis and even of $34.6 \%$ due to E. faecium has been reported, with age, comorbidity, and hospitalacquired infections as predictors of deadly courses [10]. Another analysis described a 7-day mortality of $13 \%$ and a 30 -day mortality of $25 \%$ in patients with bacteremia due to vancomycin-susceptible enterococci [8]. Prolonged healthcare exposure and increased comorbidity are associated with enterococcal bacteremia $[8,22]$. The mortality risk in vancomycin-resistant enterococci (VRE) patients is generally higher $[23,24]$.

The patient numbers with 112 E. faecalis and 141 E. faecium bacteremic isolates did not allow for a statistically meaningful analysis. From the literature, it is known that invasive procedures, surgery, chronic skin ulcers, and indwelling devices are risk factors in particular for $E$. faecalis infections [25]. Community-acquired bacteremia due to E. faecalis was shown to be associated with infective endocarditis in $25 \%$ of instances [10]. For E. faecium, in contrast, it could be demonstrated that certain clones of 
increased pathogenic potential may cause invasive disease with bacteremia [26]. In cancer patients, E. faecium bacteremia is independently associated with more severe underlying illness [27, 28].

Enterococci can lead to systemic infections if the gutblood barrier fails and if the intestinal flora is disrupted by antibiotic treatment, resulting in domination of the gut flora by enterococcal species [29]. The higher rate of mixed bacterial flora in patients with enterococcal bacteremia in this study could be due to a disturbed gut-blood barrier in severely ill patients. While $27.4 \%$ of mixed bacterial infections were observed in this study, a previous assessment suggested up to $39 \%$ bacteremia with enterococci plus other species [10]. Interestingly, in this study, typically skin-inhabiting coagulase-negative staphylococci (CNS) predominated as partners in enterococcal bacteremia, while typical gut colonizers were less frequently observed. There is a formal possibility that CNS in blood cultures result from improper skin disinfection during specimen sampling [30, 31]. However, the legally enforced annual surveillance on infectious disease data of the Rostock University Hospital demonstrates CNS rates in blood cultures at or below the average of the hospitals in this region. In addition, CNS were more frequently associated with enterococcal but not with nonenterococcal bacteremias. Therefore, the occurrence of enterococci could be interpreted as a warning sign for critical disease courses with failing gut-blood barrier. However, risk of dying due to polymicrobial bacteremia is $21 \%$ [32] and thus comparable with the general risk of dying as observed in this study.

The study has several limitations. First of all, the number of assessed samples does not allow for detailed sub-group analyses. Future studies with higher numbers of included patients should address the impact of immunosuppression of affected patients and the question whether comparable results can be achieved for patients with repeated proof of enterococci in blood culture. In addition, it would be interesting to compare healthcare-associated from community-acquired infections with enterococci, which was not analyzed by this approach.

\section{Summary}

In summary, it was shown that patients with enterococcal bacteremia exhibit a risk of dying comparable to that for $E$. coli and $S$. aureus bacteremia and an even higher morbidity as expressed by need for catecholamines and invasive ventilation. Thus, patients dying with enterococcal bacteremia are usually more severely ill than patients with nonenterococcal bloodstream infections. Together with the lack of association between typical signs of shock and death due to enterococcal bacteremia, the low pathogenic potential of enterococci in the bloodstream has to be acknowledged when compared with bacteremia due to $E$. coli and $S$. aureus. Thus, in many cases, enterococcal bacteremia could rather be an epiphenomenon of impend- ing death than one of its true causes. The higher frequency of mixed bacterial blood cultures indicating failure of the gut-blood barrier further supports this interpretation for patients with enterococcal bacteremia.

\section{Funding sources}

There was no source of funding.

\section{Conflicts of interest}

The authors declare that there are no conflicts of interest.

\section{References}

1. Ronald A: The etiology of urinary tract infection: traditional and emerging pathogens. Dis Mon 49, 71-82 (2003)

2. Megran DW: Enterococcal endocarditis. Clin Infect Dis 15, 63-71 (1992)

3. Bayer AS, Seidel JS, Yoshikawa TT, Anthony BF, Guze LB: Group D enterococcal meningitis. Clinical and therapeutic considerations with report of three cases and review of the literature. Arch Intern Med 136, 883-886 (1976)

4. Tornero E, Senneville E, Euba G, Petersdorf S, RodriguezPardo D, Lakatos B, Ferrari MC, Pilares M, Bahamonde A, Trebse R, Benito N, Sorli L, del Toro MD, Baraiaetxaburu JM, Ramos A, Riera M, Jover-Sáenz A, Palomino J, Ariza J, Soriano A; European Society Group of Infections on Artificial Implants (ESGIAI): Characteristics of prosthetic joint infections due to Enterococcus sp. and predictors of failure: a multi-national study. Clin Microbiol Infect 20, 1219-1224 (2014)

5. Mittelkötter U: Antimicrobial prophylaxis for abdominal surgery: is there a need for metronidazole? J Chemother 1, 27-34 (2001)

6. Solomkin JS, Mazuski JE, Bradley JS, Rodvold KA, Goldstein EJ, Baron EJ, O’Neill PJ, Chow AW, Dellinger EP, Eachempati SR, Gorbach S, Hilfiker M, May AK, Nathens AB, Sawyer RG, Bartlett JG: Diagnosis and management of complicated intra-abdominal infection in adults and children: guidelines by the Surgical Infection Society and the Infectious Diseases Society of America. Clin Infect Dis 50, 133-164 (2010)

7. Epstein EZ, Kugel MA: The significance of postmortem bacteriological examination: With special reference to streptococci and enterococci. J Infect Dis 44, 327-334 (1929)

8. McBride SJ, Upton A, Roberts SA: Clinical characteristics and outcomes of patients with vancomycin-susceptible $E n$ terococcus faecalis and Enterococcus faecium bacteraemia - a five-year retrospective review. Eur J Clin Microbiol Infect Dis 29, 107-114 (2010)

9. Vydra J, Shanley RM, George I, Ustun C, Smith AR, Weisdorf DJ, Young JA: Enterococcal bacteraemia is associated with increased risk of mortality in recipients of allogeneic hematopoietic stem cell transplantation. Clin Infect Dis 55, 764-770 (2012)

10. Pinholt M, Ostergaard C, Arpi M, Bruun NE, Schønheyder HC, Gradel KO, Søgaard M, Knudsen JD; Danish Collaborative Bacteraemia Network (DACOBAN): Incidence, 
clinical characteristics and 30-day mortality of enterococcal bacteraemia in Denmark 2006-2009: a populationbased cohort study. Clin Microbiol Infect 20, 145-151 (2014)

11. Kajihara T, Nakamura S, Iwanaga N, Oshima K, Takazono T, Miyazaki T, Izumikawa K, Yanagihara K, Kohno N, Kohno S: Clinical characteristics and risk factors of enterococcal infections in Nagasaki, Japan: a retrospective study. BMC Infect Dis 15, 426 (2015)

12. Glauser MP, Zanetti G, Baumgartner JD, Cohen J: Septic shock: pathogenesis. Lancet 338, 732-736 (1991)

13. Laupland KB, Church DL: Population-based epidemiology and microbiology of community-onset bloodstream infections. Clin Microbiol Rev 27, 647-664 (2014)

14. Lewis WH: The evolution of clinical sphygmomanometry. Bull N Y Acad Med 17, 871-881 (1941)

15. Top J, Willems R, Blok H, de Regt M, Jalink K, Troelstra A, Goorhuis B, Bonten M: Ecological replacement of Enterococcus faecalis by multiresistant clonal complex 17 Enterococcus faecium. Clin Microbiol Infect 13, 316-319 (2007)

16. Weisser M, Capaul S, Dangel M, Elzi L, Kuenzli E, Frei R, Widmer A: Additive effect of Enterococcus faecium on enterococcal bloodstream infections: a 14-year study in a Swiss tertiary hospital. Infect Control Hosp Epidemiol 34, 1109-1112 (2013)

17. Coombs GW, Pearson JC, Daley DA, Le T, Robinson OJ, Gottlieb T, Howden BP, Johnson PD, Bennett CM, Stinear TP, Turnidge JD; Australian Group on Antimicrobial Resistance: Molecular epidemiology of enterococcal bacteremia in Australia. J Clin Microbiol 52, 897-905 (2014)

18. Gastmeier P, Geffers C, Herrmann M, Lemmen S, Salzberger B, Seifert H, Kern W, Fätkenheuer G: Nosocomial infections and infections with multidrug-resistant pathogens - frequency and mortality. Dtsch Med Wochenschr 141, 421-426 (2016)

19. Rajkumari N, Mathur P, Thanbuana B, Sajan S, Misra MC: Magnitude of enterococcal bacteraemia in trauma patients admitted for intensive trauma care: a tertiary care experience from South Asian country. J Lab Physicians 7, 38-42 (2015)

20. Claeys KC, Zasowski EJ, Lagnf AM, Rybak MJ: Comparison of outcomes between patients with single versus multiple positive blood cultures for Enterococcus: infection versus illusion? Am J Infect Control 44, 47-49 (2016)

21. Gawryszewska I, Żabicka D, Bojarska K, Malinowska K, Hryniewicz W, Sadowy E: Invasive enterococcal infections in Poland: the current epidemiological situation. Eur J Clin Microbiol Infect Dis 35, 847-856 (2016)

22. Moses V, Jerobin J, Nair A, Sathyendara S, Balaji V, George IA, Peter JV: Enterococcal bacteraemia is associated with prolonged stay in the medical intensive care unit. J Glob Infect Dis 4, 26-30 (2012)

23. DiazGranados CA, Zimmer SM, Klein M, Jernigan JA: Comparison of mortality associated with vancomycin- resistant and vancomycin-susceptible enterococcal bloodstream infections: a meta-analysis. Clin Infect Dis 41, 327333 (2005)

24. Prematunge C, MacDougall C, Johnstone J, Adomako K, Lam F, Robertson J, Garber G. VRE and VSE bacteraemia outcomes in the era of effective VRE therapy: a systematic review and meta-analysis. Infect Control Hosp Epidemiol 37, 26-35 (2016)

25. Hayakawa K, Marchaim D, Palla M, Gudur UM, Pulluru H, Bathina P, Alshabani K, Govindavarjhulla A, Mallad A, Abbadi DR, Chowdary D, Kakarlapudi H, Guddati H, Das M, Kannekanti N, Vemuri P, Doddamani R, Mundra VR, Guddeti RR, Policherla R, Bai S, Lohithaswa S, Shashidharan SP, Chidurala S, Diviti S, Sukayogula K, Joseph M, Pogue JM, Lephart PR, Martin ET, Rybak MJ, Kaye KS: Epidemiology of vancomycin-resistant Enterococcus faecalis: a case-case-control study. Antimicrob Agents Chemother 57, 49-55 (2013)

26. Lu CL, Chuang YC, Chang HC, Chen YC, Wang JT, Chang SC: Microbiological and clinical characteristics of vancomycin-resistant Enterococcus faecium bacteraemia in Taiwan: implication of sequence type for prognosis. J Antimicrob Chemother 67, 2243-2249 (2012)

27. Conde-Estévez D, Grau S, Albanell J, Terradas R, Salvadó M, Knobel H: Clinical characteristics and outcomes of patients with vancomycin-susceptible Enterococcus faecalis and Enterococcus faecium bacteraemia in cancer patients. Eur J Clin Microbiol Infect Dis 30, 103-108 (2011)

28. Gudiol C, Ayats J, Camoez M, Domínguez MÁ, GarcíaVidal C, Bodro M, Ardanuy C, Obed M, Arnan M, Antonio $\mathrm{M}$, Carratalà $\mathrm{J}$ : Increase in bloodstream infection due to vancomycin-susceptible Enterococcus faecium in cancer patients: risk factors, molecular epidemiology and outcomes. PLoS One 2013;8:e74734 (2013)

29. Taur Y, Xavier JB, Lipuma L, Ubeda C, Goldberg J, Gobourne A, Lee YJ, Dubin KA, Socci ND, Viale A, Perales MA, Jenq RR, van den Brink MR, Pamer EG: Intestinal domination and the risk of bacteremia in patients undergoing allogeneic hematopoietic stem cell transplantation. Clin Infect Dis 55, 905-914 (2012)

30. Freeman JT, Chen LF, Sexton DJ, Anderson DJ: Blood culture contamination with Enterococci and skin organisms: implications for surveillance definitions of primary bloodstream infections. Am J Infect Control 39, 436-438 (2011)

31. Jindai K, Strerath MS, Hess T, Safdar N: Is a single positive blood culture for Enterococcus species representative of infection or contamination? Eur J Clin Microbiol Infect Dis 33, 1995-2003 (2014)

32. Reuben AG, Musher DM, Hamill RJ, Broucke I: Polymicrobial bacteraemia: clinical and microbiologic patterns. Rev Infect Dis 11, 161-183 (1989) 